This item was submitted to Loughborough's Research Repository by the author.

Items in Figshare are protected by copyright, with all rights reserved, unless otherwise indicated.

\title{
Assessment of reproducibility of thigh marker ranking during walking and landing tasks
}

PLEASE CITE THE PUBLISHED VERSION

http://dx.doi.org/10.1016/j.medengphy.2012.05.006

PUBLISHER

Elsevier / @ IPEM

VERSION

AM (Accepted Manuscript)

\section{PUBLISHER STATEMENT}

This work is made available according to the conditions of the Creative Commons Attribution-NonCommercialNoDerivatives 4.0 International (CC BY-NC-ND 4.0) licence. Full details of this licence are available at: https://creativecommons.org/licenses/by-nc-nd/4.0/

\section{LICENCE}

CC BY-NC-ND 4.0

\section{REPOSITORY RECORD}

Monnet, Tony, Arsene Thouze, Matthew T.G. Pain, and Mickael Begon. 2019. "Assessment of Reproducibility of Thigh Marker Ranking During Walking and Landing Tasks”. figshare. https://hdl.handle.net/2134/16689. 


\title{
Assessment of reproducibility of thigh marker ranking during walking and landing tasks
}

\author{
Monnet, $T^{a}$. , Thouzé, $A^{a, c}$., Pain, M.T.G ${ }^{b}$, and. Begon, M. ${ }^{c}$
}

\begin{abstract}
a Institut Pprime, UPR 3346, CNRS - Université de Poitiers - ENSMA, Bvd M\&P, Curie, BP 30179, 86962 Futuroscope Cedex, France

b School of Sport, Exercise and Health Sciences, Loughborough University, Leics LE11 3TU, UK

c Department of Kinesiology, University of Montreal, CEPSUM 2100, boul. Edouard-Montpetit C.P. 6128, succursale Centre-ville, Montréal, Québec H3C 3J7, Canada
\end{abstract}

Keywords: Biomechanics, Thigh kinematics, Ranking procedure, Soft tissue artefact,

Stereophotogrammetry, Movement analysis

\begin{abstract}
The aim of this paper is to analyse the repeatability of marker deformation and marker ranking across subjects and motor tasks. A method based on the solidification of the thigh with optimized rototranslation was applied which used 26 markers placed on the left thigh. During five trials of landing and five trials of walking for eight participants, the deformation between the actual positions of the 26 markers and the recalled positions from solidification were calculated. Markers were then sorted and ranked from the most deformed to the least deformed. Like previous studies, marker deformation found in this paper is subject and movement-dependant. The reproducibility of the marker rankings was assessed using Kendall's coefficient of concordance. Results highlighted that the marker ranking was similar between the trials of landing and between the trials of walking. Moreover, for walking and landing the rankings were consistent across the eight subjects.
\end{abstract}

\section{INTRODUCTION}

A fundamental assumption in human movement analysis is to consider human segments as rigid bodies in order to simplify the application of the laws of mechanics. Human segments are composed of bones and soft tissues and although in most instances the bones approximate rigid bodies, often the soft tissues do not. This can give rise to soft tissue artefacts (STA) due to muscular contractions, skin elasticity and wobbling masses [1] where STA are defined as skin movements relative to the underlying bone [2,3]. Researchers are continually trying to mitigate the influence of STA on the assessment of joint kinematics by analysing deformations of several marker sets [4-6]. Quantification of STA has been determined based on medical imaging [4,7], mathematical procedures $[5,6,8-11]$ or by comparison with imaging or intra-cortical pins [12]. Since methods based on stereophotogrammetry found similar STA to fluoroscopy $[5,6]$, non-invasive approaches are used more and more since they can be applied to larger populations and utilize more extensive movements without range of motion limitation [13].

Papers previously cited have proposed methods for assessing bone kinematics by reducing the STA. However, soft-tissue dynamics plays an important role, especially in joint dynamics during impacts by reducing joint loads and passively dissipating energy [14]. For example, a wobbling mass model of landing from a drop better reproduced the vertical ground reaction force than a rigid body 
model did and had lower joint forces and torques [1]. In gross motion analysis it can be more important to assess the kinematics of the whole segment than focus on the bone kinematics. Indeed the mass of the bone only represents $30 \%$ of the total mass of the thigh [15]. As a result soft tissue dynamics should play an important role in joint dynamics $[15,16]$. Given this fact marker locations that represent the best whole segment motion need to be determined in order to better estimate joint torques.

To analyse three-dimensional (3D) kinematics, local systems of coordinates (SoC) are defined from bony landmarks and joint centre locations [17] so that joint kinematics can be interpreted in anatomical terms (e.g. flexion-extension, abductionadduction and internal-external rotation). For the thigh the greater trochanter, femoral condyles and hip joint centre are the ISB recommended landmarks [18]. Using motion analysis systems, anatomical landmarks are given by either skin mounted markers or, the Calibrated Anatomical System Technique (CAST [19]). For joint centre location, much interest has been shown in the functional approach $[20,21]$. For a fully defined system both CAST and functional methods require technical markers ( $n \geq 3$ ) on each segment and locations are often chosen to minimize STA and occlusions. Only a few papers have focused on technical marker placement [4-6] even though different marker sets have been shown to result in different joint centre locations [22,23]. Indeed STA are non-homogeneous and probably subject and task dependent. Our main objective is to determine marker locations on the thigh that fit the best whole kinematics of the thigh as defined by markers on the surface. Due to specific muscular contractions and body composition, the absolute magnitude of soft tissue motion is (i) subject-specific $[2,4]$ which complicates the determination of optimal marker set for a population and (ii) task-specific [4,24]. We hypothesized that an analysis based on ranking instead of absolute deformation should provide a better insight into surface movement. Our assumptions are that the intra/inter-subject and inter-movement marker rankings are reproducible. These hypotheses were evaluated on two movements with different acceleration, namely walking and landing using a non-invasive approach on eight subjects.

\section{METHODS}

In the present study, the algorithm of Monnet et al. [25] (presented below) and recommendations of Roosen et al. [22] were applied to define the thigh as a rigid segment based upon a static configuration. Marker deformation was calculated as the Euclidean distance between the recorded marker location and its recalled location from a rigid segment assumption. The deformation was analysed in terms of absolute value and also by ranking to avoid subject-specific soft tissue range of motion confounding results across subjects.

\subsection{Equipment set up}

Eight male participants $(23.0 \pm 2.9$ years old, $178 \pm 3.7 \mathrm{~cm}, 73.6 \pm 5.6 \mathrm{~kg}$, body fat percentage $12.2 \pm 3.6$ ) took part in this study after giving informed consent in accordance with local ethical procedures. Movement data were collected at 300 $\mathrm{Hz}$ using a 10 camera Vicon system (T40, $4 \mathrm{Mpx}$ ). No signal processing was applied to the raw data. A set of 26 markers $(\varnothing 14 \mathrm{~mm})$ was methodically placed on the left thigh with double-sided tape (Fig. 1). They described five vertical lines of four to six equidistant markers: interior side of the thigh, vastus medialis, vastus lateralis, fascia lata and posterior side of the thigh. This placement was reproducible across subjects 
[26] and covered all the thigh parts that could be seen during the movements and gave a representation of the overall segment kinematics.

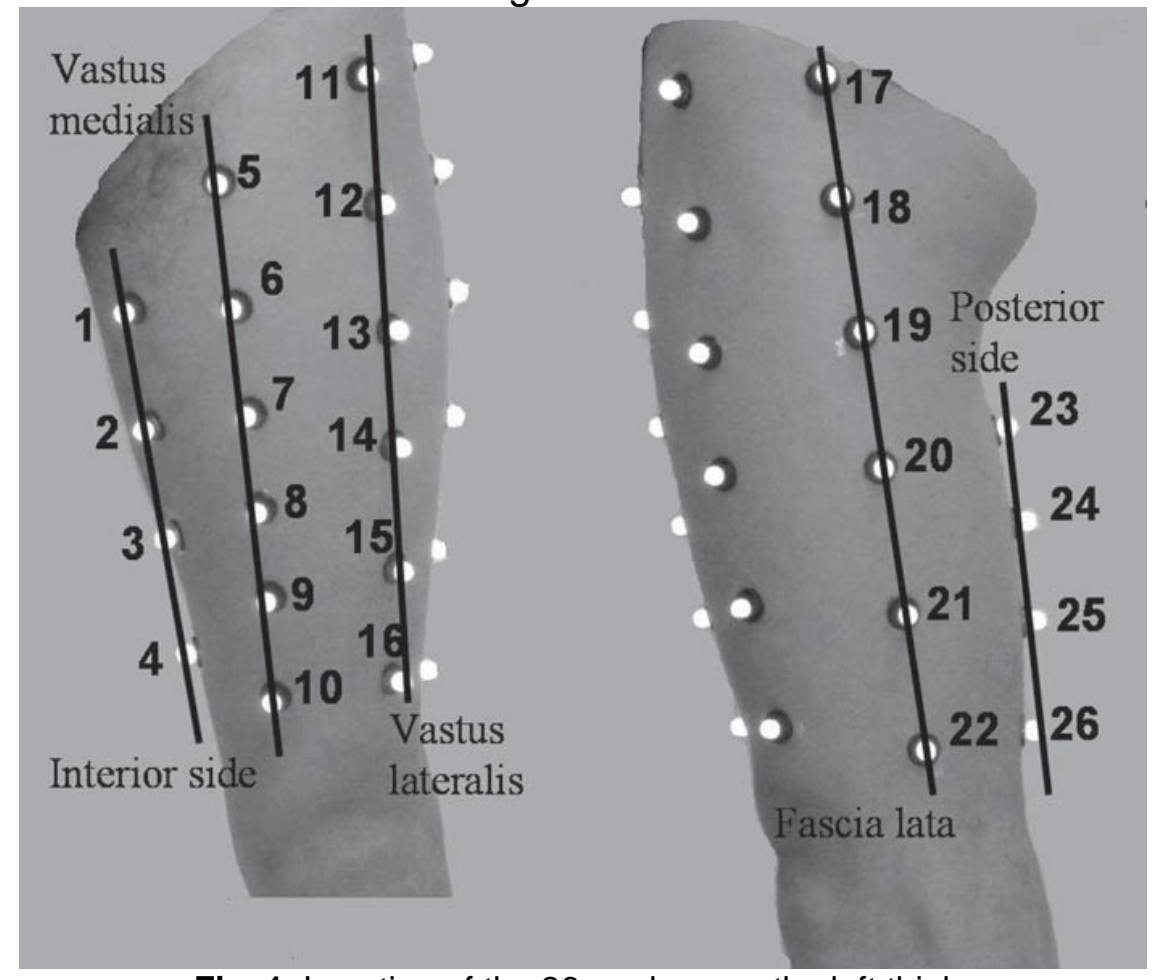

Fig. 1. Location of the 26 markers on the left thigh.

\subsection{Static acquisition}

A reference geometry for the set of markers was acquired for all subjects during a $3 \mathrm{~s}$ static anatomical posture. From this posture the local coordinates ${ }^{L} \boldsymbol{m}_{i}$ of each marker ( $i=1$ to 26 ) were calculated from its global coordinates ${ }^{G} \boldsymbol{m}_{i}$. The local frame $\mathcal{R}_{L}$ was created using all the markers ${ }^{24}$. The origin ${ }^{G} \boldsymbol{t}$ of the local frame was determined as the centroid of the 26 markers and ${ }^{G} R_{L}$ is the rotation matrix from global to local frame:

$$
\begin{gathered}
{ }^{L} \boldsymbol{m}_{\boldsymbol{i}}={ }^{G} R_{L}\left({ }^{G} \boldsymbol{m}_{i}-{ }^{G} \boldsymbol{t}\right) \\
\text { with } \\
{ }^{L} \boldsymbol{m}_{i}=\left[\begin{array}{c}
m^{m} x_{i} \\
{ }^{m} y_{i} \\
{ }^{m} z_{i}
\end{array}\right]_{\mathcal{R}_{L}} \quad{ }^{G} \boldsymbol{m}_{i}=\left[\begin{array}{l}
m \\
x_{i} \\
m_{y} y_{i} \\
{ }^{m} z_{i}
\end{array}\right]_{\mathcal{R}_{G}} \quad \text { and } \quad{ }^{G} \boldsymbol{t}=\frac{1}{n} \sum_{i=1}^{n}{ }^{G} \boldsymbol{m}_{i}
\end{gathered}
$$

The rotation matrix was calculated in each time frame using the optimization procedure described in Bouby et al. ${ }^{26}$ that involves all the markers (see appendix A). Average local coordinates calculated over the duration of the static acquisition were used as the reference position in the following sections.

\subsection{Walking and landing trials}

Subjects were asked to perform five walking trials at a self-selected speed of progression and five landing trials from a height of $0.7 \mathrm{~m}$. After marker reconstruction, a successful trial was determined as one with marker occlusions in less than $1 \%$ of frames. During each trial, the recalled positions $\left({ }^{G} \boldsymbol{r}_{i}\right)$ of the markers were calculated in the global frame using their local coordinates $\left({ }^{L} \boldsymbol{m}_{i}\right)$, previously determined during static acquisition, and the optimized rototranslation $\left(R^{\wedge}\right)$ :

$$
{ }^{G} \boldsymbol{r}_{i}={ }^{L} R_{G}{ }^{L} \boldsymbol{m}_{i}+{ }^{G} \boldsymbol{t} .
$$


Then for each marker $i$ the Euclidean distance between its actual positions $\left({ }^{G} \boldsymbol{m}_{i}\right)$ and its recalled positions ${ }^{G} \boldsymbol{r}_{i}$ was calculated at each instant of time:

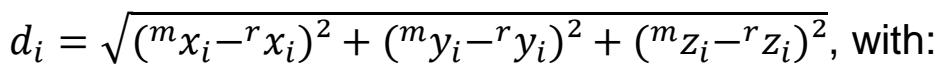

$$
\begin{aligned}
& { }^{G} \boldsymbol{r}_{i}=\left[\begin{array}{c}
{ }^{r} x_{i} \\
{ }^{r} y_{i} \\
{ }^{r} z_{i}
\end{array}\right]_{\mathcal{R}_{G}}
\end{aligned}
$$

The deformation associated with each marker was defined as the average distance $(\bar{d})$ over all frame numbers of a trial. The deformation calculated in this paper does not correspond to STA as the reference was the global segment kinematics based on all the markers and not the bone kinematics. Finally ranking procedure was performed: the first rank was accorded to the least deformed marker while the most deformed was ranked at the $26^{\text {th }}$ position.

\section{$2.4 \quad$ Statistical analysis}

A mean deformation was calculated over the five trials of each movement. Results were tested for normality with the Shapiro-Wilkinson test. Then, a three ways (movement, subject, markers) repeated measures ANOVA was used to test for significant difference $(\alpha<0.01)$ among the marker deformations, the subjects and the movement.

Kendall's coefficient of concordance $\left(W^{27}\right)$ was then calculated to assess the agreement of the marker ranking between the five trials of walking and between the five trials of landing for each subject (intra-subject reproducibility). The agreement of the marker ranking between the eight subjects during walking, landing and both movements was also assessed using Kendall's $W$ (inter-subjects reproducibility). To test $W$ for statistical significance, the $\chi^{2}$ statistic is obtained from $W$ using the formula:

$$
\chi^{2}=p(n-1) W,
$$

where $p$ is the number of observation ( 5 trials or 8 subjects) and $n(26)$ is the number of markers. The critical value of the $\chi^{2}$ distribution was predetermined at 52.62 for $\alpha=0.01$ with 25 degrees of freedom. If the value of $\chi^{2}$ equals or exceeds the critical value, the marker order is not random.

\section{RESULTS}

\subsection{Marker deformation}

Results of the three ways repeated measures ANOVA reveal that the marker deformation was subject- and movement-specific $(p<0.01)$. A post-hoc Tuckey's test was performed for each subject on landing and walking. Results of this analysis are presented for subject 1 in Figure 2a where black and gray squares indicate markers that gave the same deformation as the best and the worst markers, respectively. 

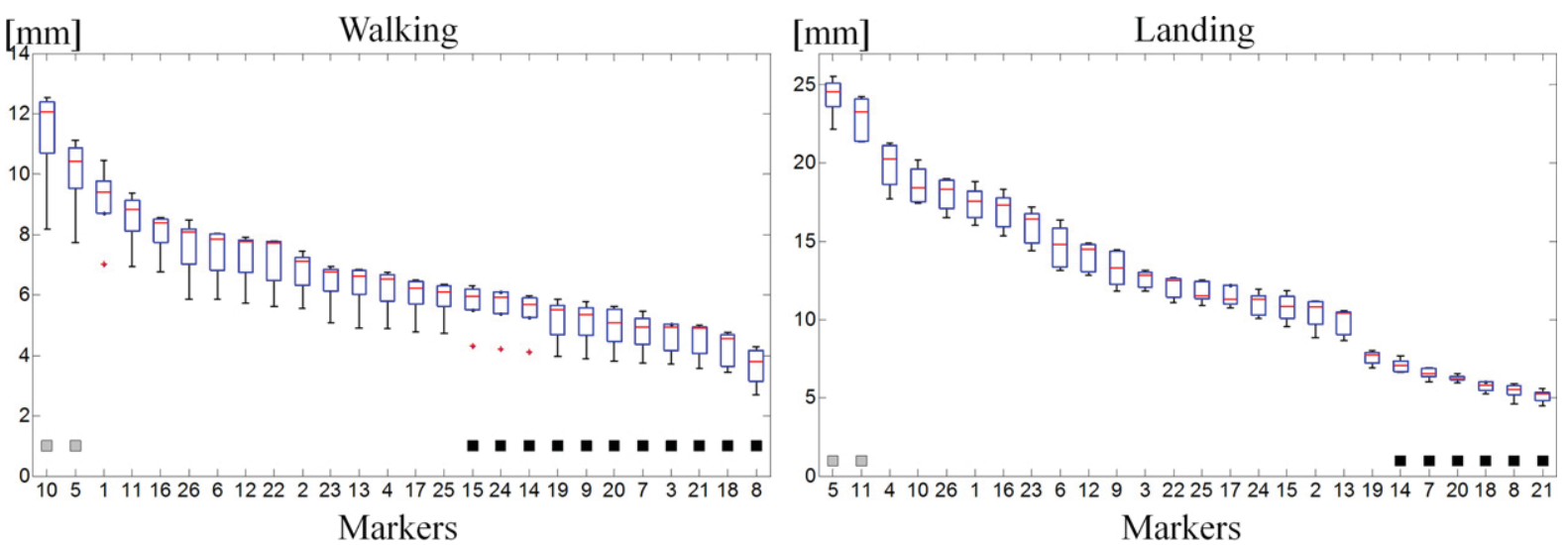

Fig. 2. (a) Marker deformations for walking and landing for subject 1. Black squares reveal markers that gave the same deformation as the least deformed and grey squares reveal markers that gave the same deformation as the most deformed.

Individual results for subjects 2 to 7 are presented as figures in the Appendix. During walking marker deformations ranged from $3.3 \pm 0.3 \mathrm{~mm}$ for subject 2, marker 8 (mid-part of the vastus medialis) to $16.2 \pm 1.5 \mathrm{~mm}$ for subject 3,marker 5 (upper part of the vastus medialis). During landing marker deformations ranged from $2.6 \pm 0.3 \mathrm{~mm}$ for subject 2, marker 21 (third lower part of the fascia lata) to $28.1 \pm 2.8 \mathrm{~mm}$ also measured for subject 3 , marker 5 . A summary of these results is reported in Fig. 3 where only black and gray squares -revealing markers that gave the same deformation as the best and the worst markers respectively- are reported. During the 16 movements, walking and landing for the 8 subjects, marker 5 is the most deformed marker fifteen times, whereas markers 8 and 20 are always among the least deformed markers.
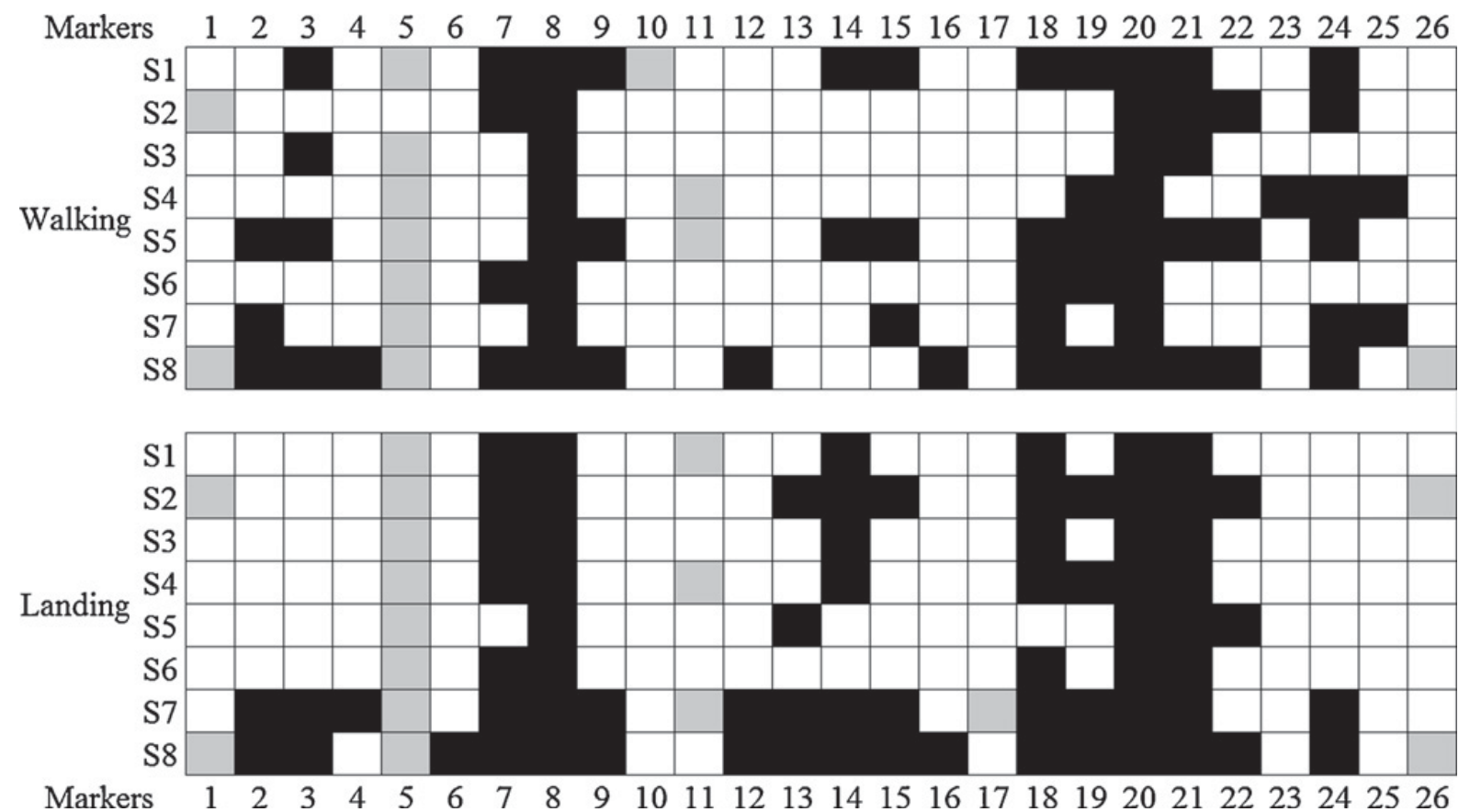

$\begin{array}{lllllllllllllllllllllllllllllllll}\text { Markers } & 1 & 2 & 3 & 4 & 5 & 6 & 7 & 8 & 9 & 10 & 11 & 12 & 13 & 14 & 15 & 16 & 17 & 18 & 19 & 20 & 21 & 22 & 23 & 24 & 25 & 26\end{array}$ Fig. 3. Summary of the results for the marker deformations. For each subject and movement, black squares indicate markers that gave the same deformation as the best one and grey squares indicate markers that gave the same deformation as the worst one.

\subsection{Marker ranking}

Marker ranking is presented in Figure 4 using grayscale encoding. 


\section{Markers}

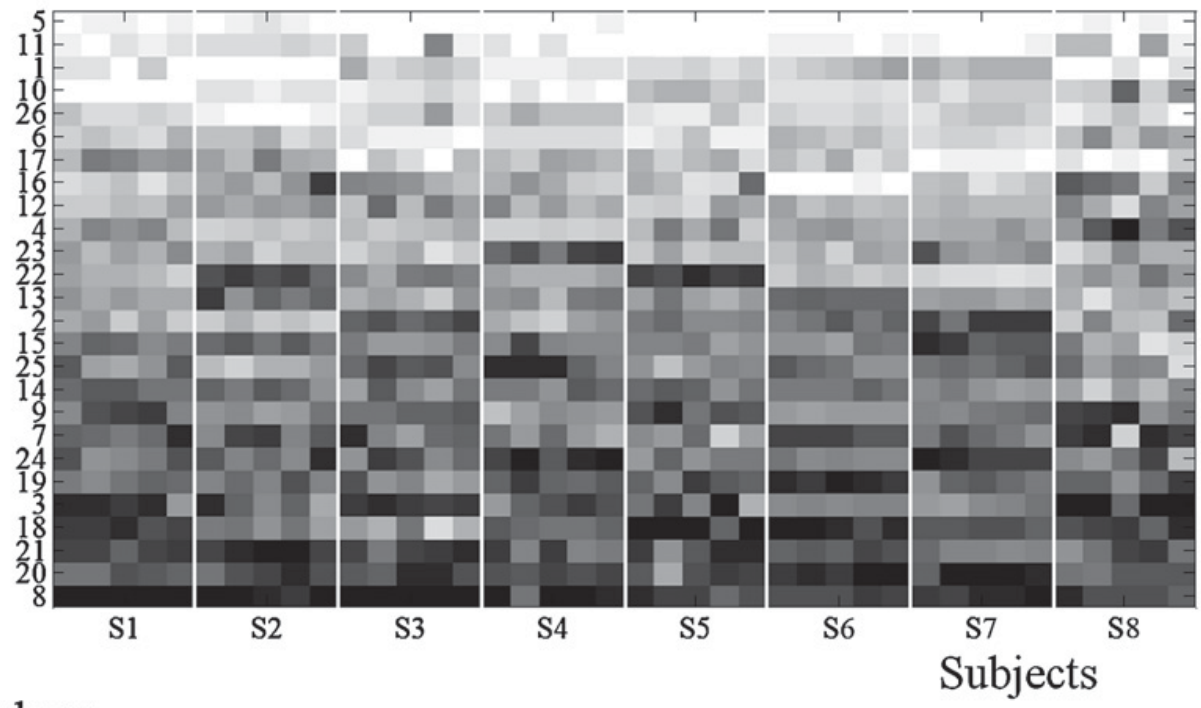

a

Last rank

Markers

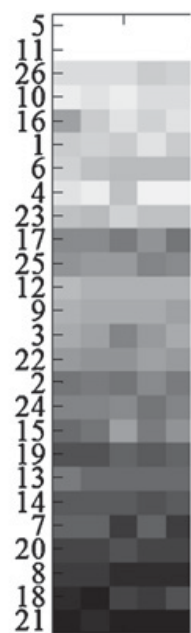

S1

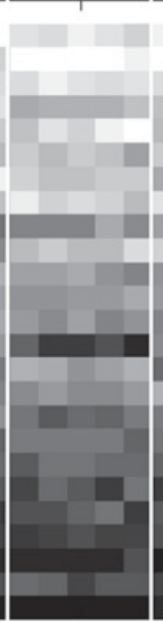

S2

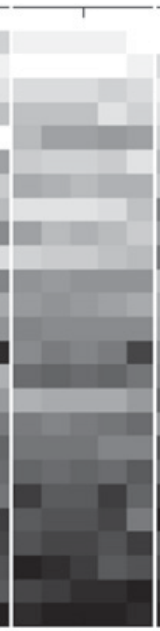

S3

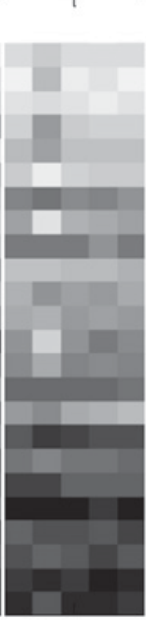

S4

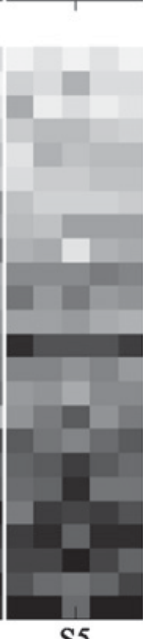

S5

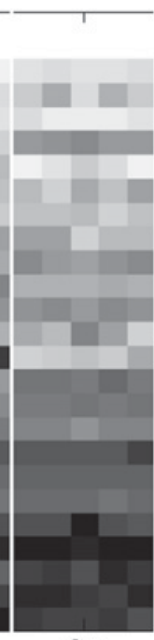

S6

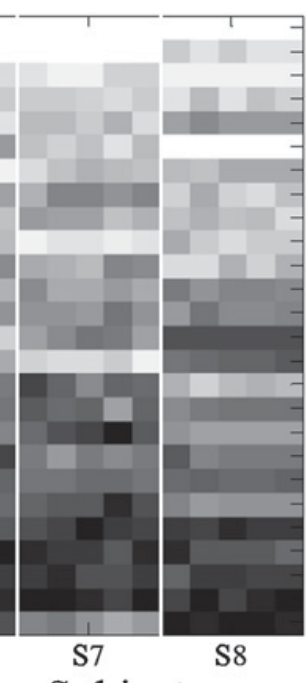

Subjects first rank

b

Fig. 4. Greyscale coded grids for the results of the ranking procedure of all the subjects during walking (a) and landing (b).

Kendall coefficient of concordance was calculated on the ranking of the five walking trials and landing trials for each subject to test the intra-subject reproducibility. In walking trials, since the value of $\chi^{2}$ exceed the critical value $(\mathrm{W}>0.75, \mathrm{p}<0.01)$ for all subjects, the marker ranking was significantly similar for each subject (Figure 5). The same result was found for landing trials $(W>0.94)$. While the inter-subject reproducibility was lower than intra-subject reproducibility in the three case (Figure 5), the rankings were significantly reproduced when considering all the subjects with Kendall's coefficient of $W=0.69, W=0.83$ and $W=0.72$ for walking, landing and their combination, respectively. 


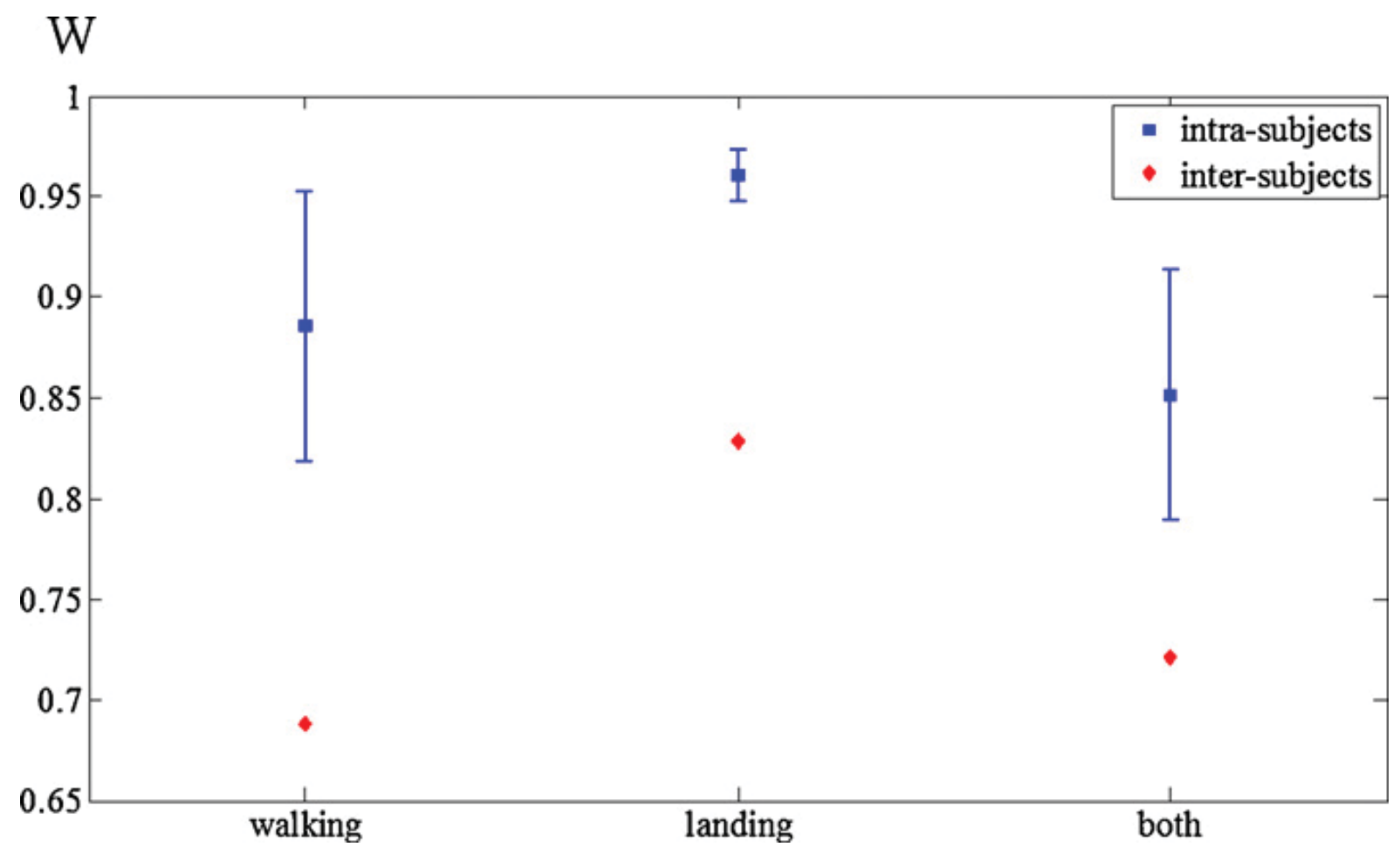

Fig. 5. Kendall coefficients of concordance according to the movements. Squaresdenote intrasubjects coefficient and triangles denote inter-subjects coefficient.

\section{Discussion}

This study aimed at assessing thigh marker deformation and marker ranking reproducibility during walking and landing. For each trial and each subject, the markers were ranked according to their average deformation over a trial with respect to an anatomical posture. Like previous studies, figures $2 \mathrm{a}-2 \mathrm{~h}$ highlight that marker deformation is subject and movement-dependant ${ }^{2,4,23}$. Nevertheless, markers 5 and 11 placed on the upper anterior face of the thigh appear as the most deformed markers most of the time, whereas markers 8 (mid part of the front thigh), 18, 20, 21 (fascia lata) are always among the least deformed ones. This observation is corroborated by using the Kendall's coefficient of concordance that highlighted that both the intra-subject ranking and the inter-subject ranking of the markers were consistent for landing and walking.

\subsection{Marker deformation}

The deformations obtained in the present study ranged from $2.6 \pm 0.3 \mathrm{~mm}$ to $28.1 \pm 2.8 \mathrm{~mm}$. In a similar study, Camomilla et al. ${ }^{5}$ reported values of soft tissue displacement from 2.5 to $23 \mathrm{~mm}$ during step up/down motor tasks, Gao et al. ${ }^{13}$ reported a range of inter-marker movement up to $19.1 \mathrm{~mm}$ and Sati et al. ${ }^{7}$ reported skin-bone movement from 2 to $17 \mathrm{~mm}$ specifically at the knee during knee flexion. The deformation obtained in the present study is not STA but corresponds to the difference between reference kinematics derived from all markers and individual marker motion. STA determined using invasive approaches such as intra-cortical pins has been found to be up to $20 \mathrm{~mm}^{23}$. As previously described, ${ }^{5,6,28}$ results obtained using various solidification procedures generally provide similar kinematics to that calculated from intra-cortical pins or invasive imaging. Intra-cortical pins can severely limit the realistic quantification of soft tissue artefact during daily living 
activities $^{4,12}$, and movement used in this paper - landing - wouldn't be analysed using MRI because it is limited to static or quasi-static investigations ${ }^{12}$.

\subsection{Deformation versus ranking}

This paper highlights the interest of using marker ranking instead of just their deformation. Marker deformation is subject- and movement-specific due to various morphotypes, range of motions and ground reaction force intensity, especially during landing $^{1}$. Our first finding was that there was intra-subject and inter-subject reproducibility of the marker ranking in walking and landing trials. The ranking is an original approach to select a minimal marker set describing the best surface movement for walking and landing or other motions.

When considering the absolute deformation for subject 8 for example, we could not distinguish between the fourteen less deformed markers for walking and between the seventeen less deformed markers for landing due to high variability between trials. On the other hand, there is a maximum of three markers for all the subjects that are most deformed compared to all other markers for each movement. Using absolute marker deformation the most deformed surface areas could be defined but it was more difficult to define the less deformed surface areas for some subjects.

\subsection{Marker locations}

The marker positions are the inputs to the joint kinematic calculation that requires the estimation of the joint centre locations using a functional method ${ }^{2}$ and the calculation of the rotation matrices. Both steps are highly sensitive to marker deformation $^{21}$. For describing the thigh kinematics, markers are commonly located on three landmarks, namely the greater trochanter and the femoral condyles ${ }^{17}$. However, the surface areas close to these anatomical landmarks exhibited deformations of up to $12 \mathrm{~mm}$ in our study, reinforcing previous findings that these may not be good locations ${ }^{12,16,23}$. For the determination of the surface thigh kinematics, the position and orientation of the whole segment should be determined from markers placed on the mid-thigh associated with an anatomical calibration $\left(\mathrm{CAST}^{18}\right)$ of the landmarks. This CAST procedure involves technical markers and is based on the assumption of rigid segments. According to the marker location, this assumption is not always respected but the most appropriate locations could be determined using our approach combined with radiographics techniques. The knowledge of STA is important to not only assess the accurate kinematics of the skeleton but to other applications, especially for inverse dynamics, where the wobbling masses motion needs to be considered as well ${ }^{29}$.

At least three markers are needed to calculate the rotation matrix but increasing the number of markers improves the accuracy ${ }^{30}$ and allows for more repeatable anatomical frame orientation ${ }^{31}$. Moreover the precision of the hip joint centre location using a functional method is increased by using more than three markers ${ }^{21}$. The present results could help in determining the best locations for these extra-markers when no reference to bone kinematics is needed. Moreover, the approach presented here is non invasive and can be applied to several situations where intracortical pins or Roentgen photogrammetry would be impossible or to nonhomogoneity population to account for individual subjects differences ${ }^{32}$. Nevertheless, the method presented here does not evaluate surface deformation with reference to underlying bone kinematics and so does not allow clinical recommendations about marker placement to be made. 
To conclude, considering marker ranking instead of marker absolute deformation allows us to determine a minimal marker set considering whole segmental kinematics for human locomotion analysis (walking and landing in this study). For eight subjects, marker ranking was similar between five trials and the ranking was reproducible across subjects. Similarly to previous papers, the worst marker locations were located near the hip and knee joint centres. From this analysis using agreement of the marker ranking instead of the absolute deformation, markers put on the external side of the thigh and on the mid-front thigh are the well ranked across subjects and movements.

\section{Acknowledgments}

This study was supported by a Poitou-Charentes regional grant (Contrat de Projet Etat Région Axe B "Savoir, Images Société") and by the Natural Sciences and Engineering Research Council of Canada.

\section{References}

[1] Pain M.T.G. and Challis J.H., The influence of soft tissue movement on ground reaction forces, joint torques and joint reaction forces in drop landings, JoB, 2006, 39, pp. 119-124.

[2] Leardini A., Chiari A., Della-Croce U. and Cappozzo A., Human movement analysis using stereophotogrammetry Part 3. Soft tissue artifact assessment and compensation, G\&P, 2005, 21, pp. 212-225.

[3] Cereatti A., Della-Corce U. and Cappozzo A., Reconstruction of skeletal movement using skin markers: comparative assessment of bone pose estimators, $\mathrm{J}$. Neuro. Rehab., 2006, 23, pp. 3-7.

[4] Stagni R., Fantozzi S., Cappello A. and Leardini A., Quantification of soft tissue artefact in motion analysis by combining $3 \mathrm{~d}$ fluoroscopy and stereophotogrammetry: a study on two subjects, Clin. Biomech., 2005, 20, pp. 320329.

[5] Camomilla V., Donati M., Stagni R. and Cappozzo A., Non-invasive assessment of superficial soft tissue local displacements during movement: A feasibility study, JoB, 2009, 42, pp. 931-937.

[6] Peters A., Sangeux M., Morris M.E. and Baker R., Determination of the optimal locations of surface-mounted markers on the tibial segment, G\&P, 2009, 29, pp. $42-48$.

[7] Sati A., de Guise J.A., Larouche S. and Drouin G., Quantitative assessment of skin-bone movement at the knee, The Knee, 1996, 3, pp. 121-138.

[8] Cappozzo A., Cappello A., Della-Croce U. and Pensalfini F., Surface-Marker Cluster Design Criteria for 3-D Bone Movement Reconstruction, IEEE Trans. Biom. Eng., 1997, 44, pp 1165-1174.

[9] Lucchetti L., Cappozzo A., Cappello A. and Della-Croce U., Skin movement artefact assessment and compensation in the estimation of knee-joint kinematics, JoB, 1998, 31, pp. 977-984.

[10] Chèze L., Fregly B.J. and Dimnet J., A solidification procedure to facilitate kinematic analyses based on video system data, JoB, 1995, 28, pp. 879—884.

[11] Lu T.W. and O'Connor J., Bone position estimation from skin marker coordinates using global optimisation with joint constraints, JoB, 1999, 32, pp. 129_ 134 ,. 
[12] Peters A., Galna B., Sangeux M., Morris M.E. and Baker R., Quantification of soft tissue artifact in lower limb human motion analysis: a systematic review, G\&P, 2010, 31, pp. 1-8.

[13] Gao B. and Zheng N.N., Investigation of soft tissue movement during level walking: Translations and rotations of skin markers, JoB, 2008, 41, pp. 3189-3195. [14] Gruber K., Ruder H., Denoth J. and Schneider K., A comparative study of impact dynamics: wobbling mass model versus rigid body models, JoB, 1998, 31, pp439-444.

[15] Tsai T-Y., Lu T-W., Kuo M-Y. and Lin C-C, Effects of soft tissue artifacts on the calculated kinematics and kinetics of the knee during stair-ascent, JoB, 2011, 44, pp 1182-1188.

[16] Cappozzo A., Catani F., Leardini A., Benedetti M.G. and Della-Croce U., Position and orientation in space of bones during movement: experimental artefacts, Clin. Biomech., 1996, 11, pp. 90-100.

[17] Wu G., Siegler S., Allard P., Kirtley C., Leardini A., Rosenbaum D., Whittle M., D'Lima D.D., Cristofolini L., Witte H., Schmid O. and Stokes I., ISB recommendation on definitions of joint coordinate system of various joints for the reporting of human joint motion--part 1: ankle, hip, and spine, JoB, 2002, 35, pp. 543-548.

[18] Cappello A., Cappozzo A., La Palombara P.F., Lucchetti L. and Leardini L., Multiple anatomical landmark calibration for optimal bone pose estimation, Hum.

Mov. Sci., 1997, 16, pp. 259-274.

[19] Leardini A., Cappozzo A., Catani F., Toksvig-Larsen S., Petitto A., Sforza V., Cassanelli G. and Giannini S., Validation of a functional method for the estimation of hip joint centre location, JoB, 1999, 32, pp. 99-103.

[20] Halvorsen K., Biais compensated least square estimate of the center of rotation, JoB, 2003, 36, pp. 999-1008.

[21] Roosen A., Pain M.T.G. and Begon M., Limitations of functionally determined joint centres for analysis of athletic human movement: A case study of the upper limb, J. App. Biomech., 2009, 25, pp. 281-292.

[22] Ehrig R. M., Heller M. O., Kratzenstein S., Duda G.N., Trepczynski A. and Taylor W.R., The SCoRE residual: A quality index to assess the accuracy of joint estimations, JoB, 2011, 44, pp 1400-1404.

[23] Fuller J., Liu L.J., Murphy M.C. and Mann R.W., A comparison of lowerextremity skeletal kinematics measured using skin- and pin-mounted markers, Hum. Mov. Sci., 1997, 16, pp. 219-242.

[24] Monnet T., Begon M., Pain M.T.G. and Colloud F., Iterative solidification procedure to determine the optimal marker location, 10th international symposium on the 3D Analysis of Human Movement, Amsterdam 28-31 October 2008.

[25] Thouzé A., Gailhard M., Cotton R. and Begon M., Implications of marker sizes during gait analysis, 14th biannual Conference of Canadian Society of Biomechanics, 2010.

[26] Bouby C., Fortuné D., Vallée C. and Pietraszkiewicz W., Direct determination of the rotation in the polar decomposition of the deformation gradient by maximizing a rayleigh quotient, Zeit. Ang. Math. Mech., 2005, 85, pp. 155-162.

[27] Kendall M.G., and Babington-Smith B., The Problem of $m$ Rankings, The Ann. Math. Stat., 1936, 10, pp. 275-287.

[28] Andriacchi T., Alexander E., Toney M., Dyrbry C. and Sum J., A point cluster method for in vivo motion analysis: applied to a study of knee kinematics, $\mathrm{J}$.

Biomech. Eng., 1998, 120, pp. 743-749. 
[29] Challis J.H. and Pain M.T.G., Soft tissue motion influences skeletal loads during impacts, Exe. Sport Sci. Rev., 2008, 36, pp. 71-75.

[30] Challis J.H., A procedure for determining rigid body transformation parameters, JoB, 1995, 28, pp. 733-737.

[31] Della-Croce U., Camomilla V., Leardini A. and Cappozzo A., Femoral anatomical frame: assessment of various definitions, Med. Eng. Phys., 2003, 25, pp. 425-431.

[32] Reinschmidt C., Bogert A., Lundberg A., Nigg B., Murphy N., Stacoff A. and Stano A., Tibiofemoral and tibiocalcaneal motion during walking: external vs. skeletal markers, G\&P, 1997, 6, pp. 98-109. 


\section{Appendix}

Appendix A. Rotation matrix optimization

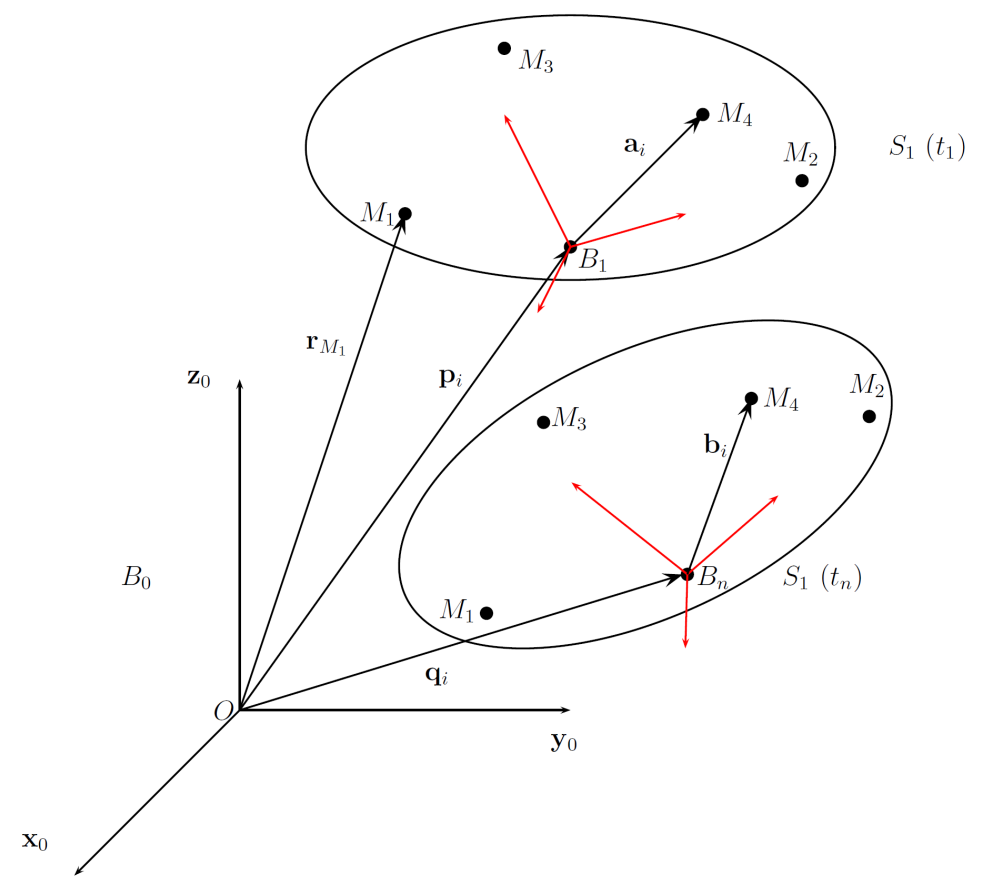

Fig. 1. Vector definition for one segment-embedded reference at two times $\left(t_{1}\right.$ and $\left.t_{n}\right)$.

All the notations refer to Fig. 1. The first step is to calculate the rotation of segment $S_{1}$ at time $t_{1}$ with respect to $B_{0}\left({ }_{0}^{t_{1}} R\right)$. The rotation matrix ${ }_{0}^{t_{1}} R$ between $B_{1}$ and $B_{0}$ is:

$$
{ }_{0}^{t_{1}} R=\left[\begin{array}{lll}
\mathbf{x} & \mathbf{y} & \mathbf{z}
\end{array}\right]
$$

where

$$
\begin{aligned}
& \mathbf{x}=\frac{\mathbf{r}_{M_{2}}\left(t_{1}\right)-\mathbf{r}_{M_{1}}\left(t_{1}\right)}{\left\|\mathbf{r}_{M_{2}}\left(t_{1}\right)-\mathbf{r}_{M_{1}}\left(t_{1}\right)\right\|}, \\
& \mathbf{z}=\mathbf{x} \times \frac{\mathbf{r}_{M_{3}}\left(t_{1}\right)-\mathbf{r}_{M_{1}}\left(t_{1}\right)}{\left\|\mathbf{r}_{M_{3}}\left(t_{1}\right)-\mathbf{r}_{M_{1}}\left(t_{1}\right)\right\|},
\end{aligned}
$$

and 


$$
\mathbf{y}=\mathbf{z} \times \mathbf{x}
$$

In the second step, the rotation matrix $R$ is the rotation of the segment $S_{1}$ from $t_{n}$ to $t_{1}: R={ }_{t_{1}}^{t_{n}} R$. The rotation matrix of the body is such that $\mathbf{p}_{i}=R \mathbf{q}_{i}+\mathbf{v}$ and $\mathbf{a}_{i}=R \mathbf{b}_{i}$. $R$ can be found by maximizing the following function (?):

$$
\operatorname{tr}\left[\frac{1}{n} \sum_{i=1}^{n}\left(\mathbf{b}_{i} \mathbf{a}_{i}^{T}\right) R\right]=\operatorname{tr}\left(C^{T} R\right),
$$

(tr represents the trace operator) with

$$
\begin{aligned}
C & =\frac{1}{n} \sum_{i=1}^{n}\left(\mathbf{b}_{i} \mathbf{a}_{i}^{T}\right), \\
\mathbf{a}_{i} & =\mathbf{r}_{M_{i}}-\frac{1}{n} \sum_{i=1}^{n} \mathbf{r}_{M_{i}}
\end{aligned}
$$

and

$$
\mathbf{b}_{i}=\mathbf{r}_{M_{i}}-\frac{1}{n} \sum_{i=1}^{n} \mathbf{b}_{M_{i}}
$$

In the present study, a procedure different to that proposed in ? is implemented for calculating $R$ without use of the square root or inverse operations based on the work of ?. Firstly, the symmetric part of $C$ and the quadrivector w associated to the skew symmetric part of $\mathrm{C}$ are computed as

$$
S=\frac{1}{2}\left(C+C^{T}\right)
$$

and

$$
j(\mathbf{w})=C-C^{T},
$$

where $j(\mathbf{w})$ is the skew-symmetric mapping defined by: $j(\mathbf{w}) \mathbf{v}=\mathbf{w} \times \mathbf{v}$.

To the $3 \times 3$ matrix $C$ is associated the $4 \times 4$ matrix $Q$,

$$
Q=\left[\begin{array}{cc}
2 S-[\operatorname{tr}(S)] \mathbb{1}_{3} & \mathbf{w} \\
\mathbf{w}^{T} & \operatorname{tr}(S)
\end{array}\right]
$$


Any rotation can be expressed by the formula:

$$
R=\left(m_{0}^{2}-\mathbf{m} \cdot \mathbf{m}\right) \mathbb{1}_{3}+2 \mathbf{m} \cdot \mathbf{m}^{T}+2 m_{0}[j(\mathbf{m})],
$$

with $\mathbf{m} . \mathbf{m}+m_{0}^{2}=1$ and $m_{0}>0\left(\mathbb{1}_{3}\right.$ is a $3 \times 3$ identity matrix $)$.

To the rotation $R$ is associated the quadrivector $\mathbf{Y}(?)$ :

$$
\mathbf{Y}=\left[\begin{array}{l}
\mathbf{m} \\
m_{0}
\end{array}\right]
$$

and

With the association of $Q$ and $Y$ to $C$ and $R$ the expression for $\operatorname{tr}\left(C^{T} R\right)$ in equation (5) becomes:

$$
\operatorname{tr}\left(C^{T} R\right)=\mathbf{Y} \cdot Q \mathbf{Y}
$$

The problem formulated in equation (5) is now to maximize:

$$
\phi(\mathbf{Y})=\frac{\mathbf{Y} \cdot Q \mathbf{Y}}{\mathbf{Y} . \mathbf{Y}}
$$

The conjugate gradient iterative algorithm is then applied (?):

- Initialise $\mathbf{Y}_{0}, \mathbf{W}_{0}=\mathbf{G}_{0}=(\operatorname{grad} \phi)\left(\mathbf{Y}_{0}\right)$

- While $\mathbf{G}_{\mathbf{i}}>\epsilon$

- Search for $\mu_{i}$ maximizing $\phi\left(\mathbf{Y}_{i}+\mu \mathbf{W}_{i}\right)$

- Compute $\mathbf{Y}_{i+1}=\mathbf{Y}_{i}+\mu_{i} \mathbf{W}_{i}$

- Compute $\mathbf{G}_{i+1}=(\operatorname{grad} \phi)\left(\mathbf{Y}_{i+1}\right)$

- Compute $\lambda_{i+1}=\frac{\mathbf{G}_{i+1} \cdot\left(\mathbf{G}_{i+1}-\mathbf{G}_{i}\right)}{\mathbf{G}_{i} \cdot \mathbf{G}_{i}}$

- Determine the new search direction $\mathbf{W}_{i+1}=\mathbf{G}_{i+1}+\lambda_{i+1} \mathbf{W}_{i}$

- end

In this algorithm, $\mathbf{G}$ and $\mu$ are calculated by the formulae:

$$
G=\frac{2}{\mathbf{Y} . \mathbf{Y}}[Q \mathbf{Y}-\phi(\mathbf{Y}) \mathbf{Y}]
$$

and 


$$
\mu=\frac{-\beta-\sqrt{\Delta}}{2 \alpha}
$$

In these formulae,

$$
\begin{aligned}
& \alpha=(\mathbf{Y} . \mathbf{W})(Q \mathbf{W} \cdot \mathbf{W})-(Q \mathbf{Y} \cdot \mathbf{W})(\mathbf{W} \cdot \mathbf{W}), \\
& \beta=(\mathbf{Y} . \mathbf{Y})(Q \mathbf{W} \cdot \mathbf{W})-(\mathbf{Y} . Q \mathbf{Y})(\mathbf{W} \cdot \mathbf{W}),
\end{aligned}
$$

and

$$
\begin{aligned}
\Delta= & \left(1-\frac{(\mathbf{Y} . \mathbf{W})^{2}}{(\mathbf{Y} . \mathbf{Y})(\mathbf{W} . \mathbf{W})}\right) \beta^{2}+4(\mathbf{Y} . \mathbf{Y})(\mathbf{W} . \mathbf{W}) \\
& {\left[Q \mathbf{Y} \cdot \mathbf{W}-\frac{(\mathbf{Y} . \mathbf{W})}{(\mathbf{Y} . \mathbf{Y})(\mathbf{W} \cdot \mathbf{W})} \frac{(\mathbf{Y} . \mathbf{Y})(Q \mathbf{W} \cdot \mathbf{W})+(\mathbf{W} . \mathbf{W})(Q \mathbf{Y} . \mathbf{Y})}{2}\right]^{2} }
\end{aligned}
$$

The rotation matrix $t_{t_{1}}^{t_{n}} R$ is then calculated from the solution (the quadrivector $\mathbf{Y})$ of the gradient algorithm with equation (12).

In a third step, at each instant of time the optimized rotation matrix ${ }_{0}^{t_{n}} R$ between $B_{n}$ and $B_{0}$ is:

$$
{ }_{0}^{t_{n}} R={ }_{0}^{t_{1}} R . \quad{ }_{t_{1}}^{t_{n}} R
$$

The translation vector is defined as the mean vector of all the markers fixed on the segment:

$$
\mathbf{t}=\frac{1}{n} \sum_{i=1}^{n} \mathbf{r}_{M_{i}}
$$

\section{References}

Bouby C, Fortuné D, Vallée C, Pietraszkiewicz W. 2005. Direct determination of the rotation in the polar decomposition of the deformation gradient by maximizing a rayleigh quotient. Zeit Ang Math Mech. 85(3):155-162.

Brousse P. 1988. Optimization in mechanics : problems and methods. North Holland Editor, Amsterdam. 
Challis JH. 1995. A procedure for determining rigid body transformation parameters. J Biomech. 28(6):733-737.

Souriau J. 1964. Géométrie et relativité. Editions Hermann, Paris. 


\section{Appendix B.}

See Figs. B1-B7.
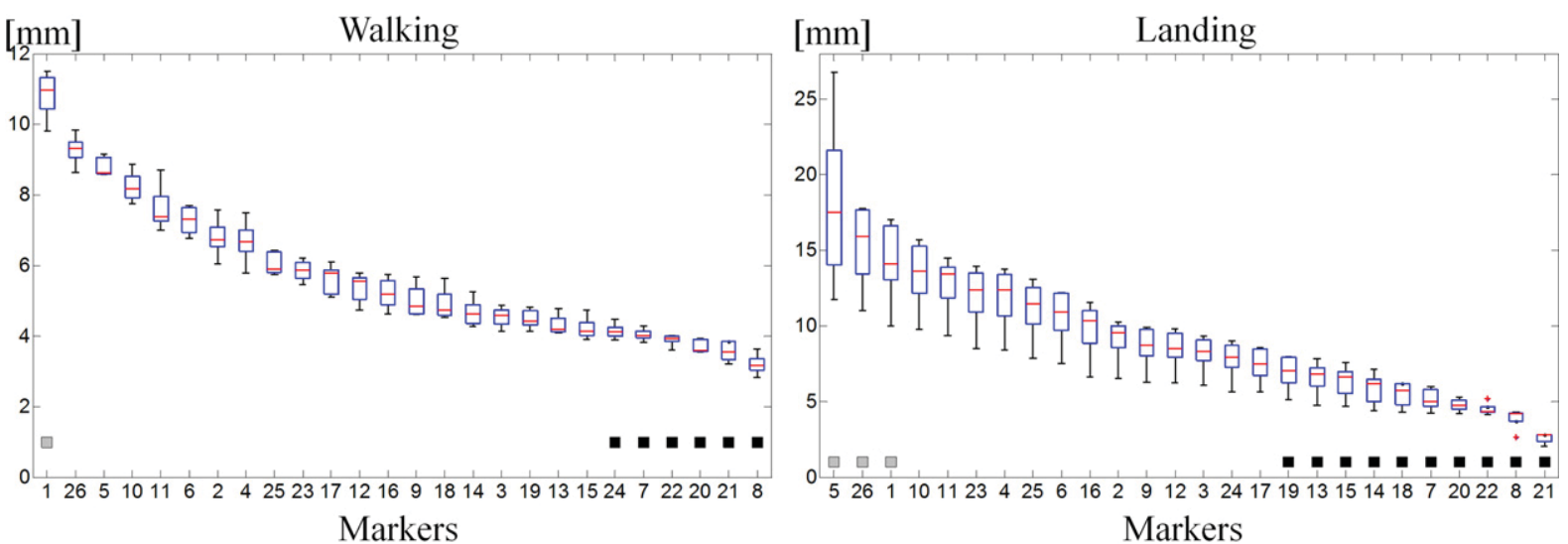

Fig. B1. Marker deformations for walking and landing for subject 2. Black squares reveal markers that gave the same deformation as the least deformed and grey squares reveal markers that gave the same deformation as the most deformed.

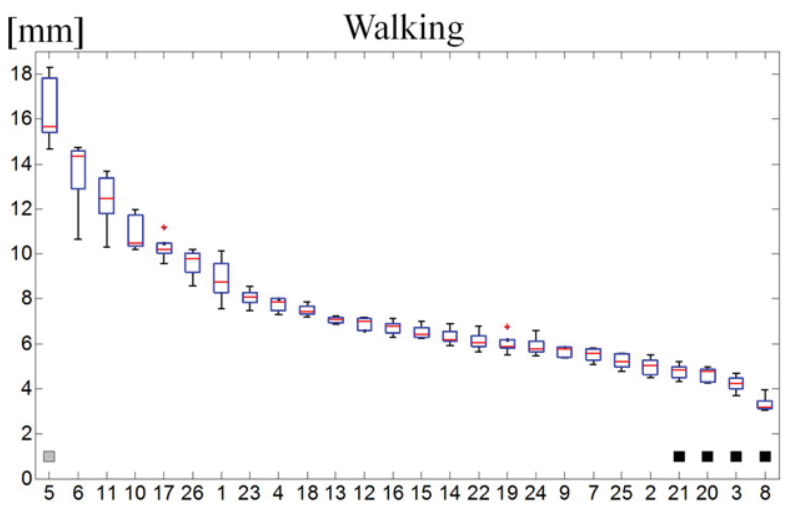

Markers

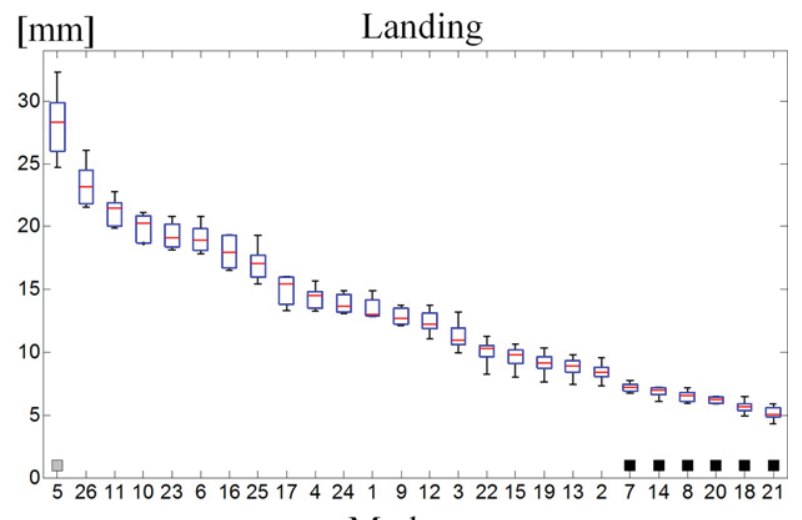

Markers

Fig. B2. Marker deformations for walking and landing for subject 3. Black squares reveal markers that gave the same deformation as the least deformed and grey squares reveal markers that gave the same deformation as the most deformed. 


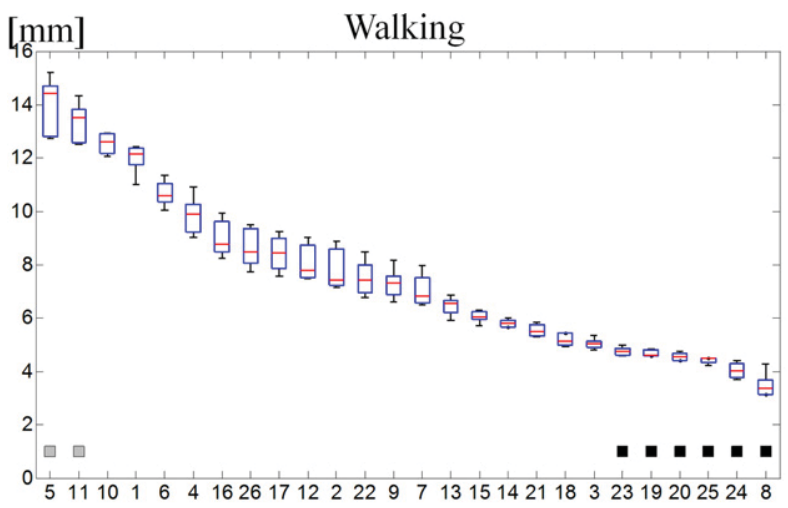

$[\mathrm{mm}] \quad$ Landing

Markers
Fig. B3. Marker deformations for walking and landing for subject 4. Black squares reveal markers that

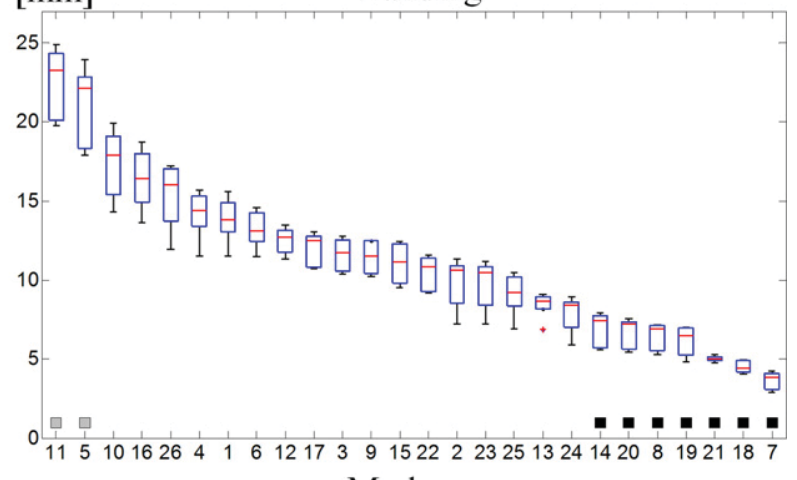
gave the same deformation as the least deformed and grey squares reveal markers that gave the same deformation as the most deformed.
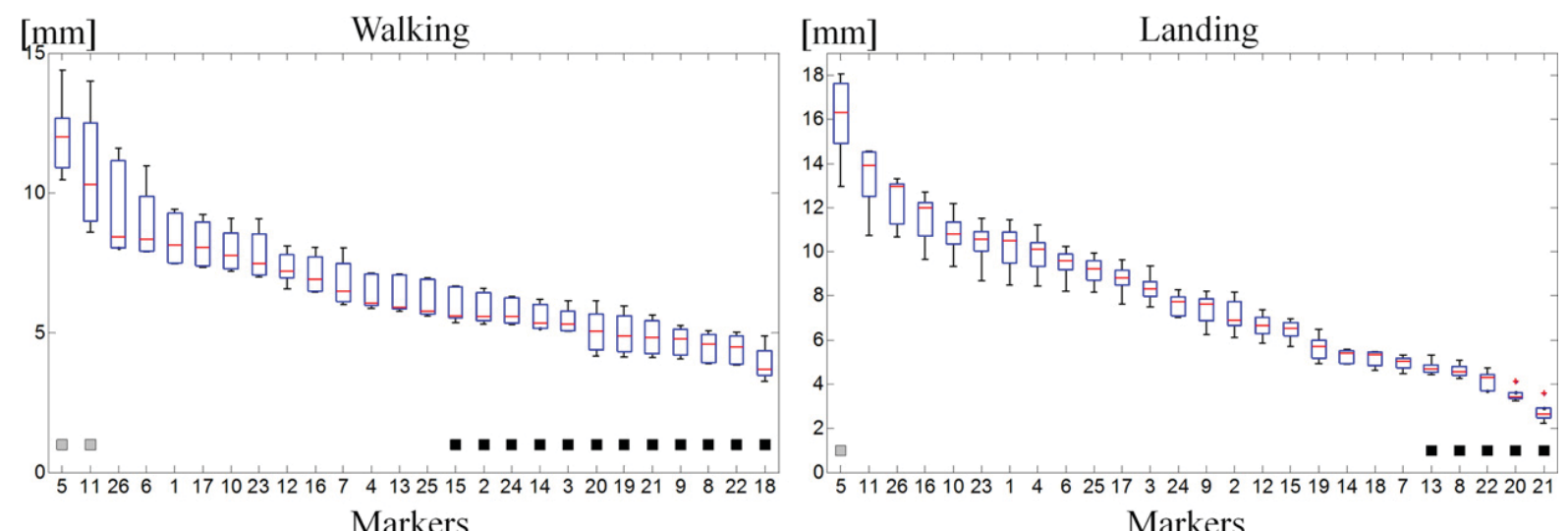

Fig. B4. Marker deformations for walking and landing for subject 5. Black squares reveal markers that gave the same deformation as the least deformed and grey squares reveal markers that gave the same deformation as the most deformed.
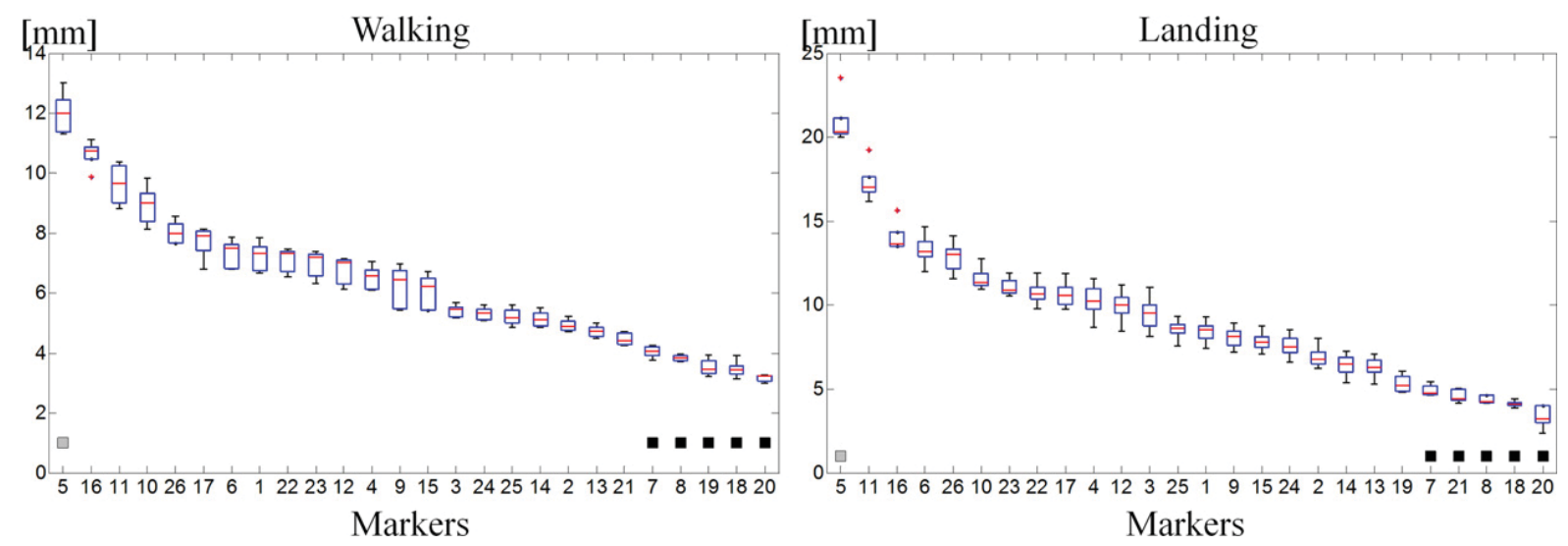

Fig. B5. Marker deformations for walking and landing for subject 6 . Black squares reveal markers that gave the same deformation as the least deformed and grey squares reveal markers that gave the same deformation as the most deformed. 


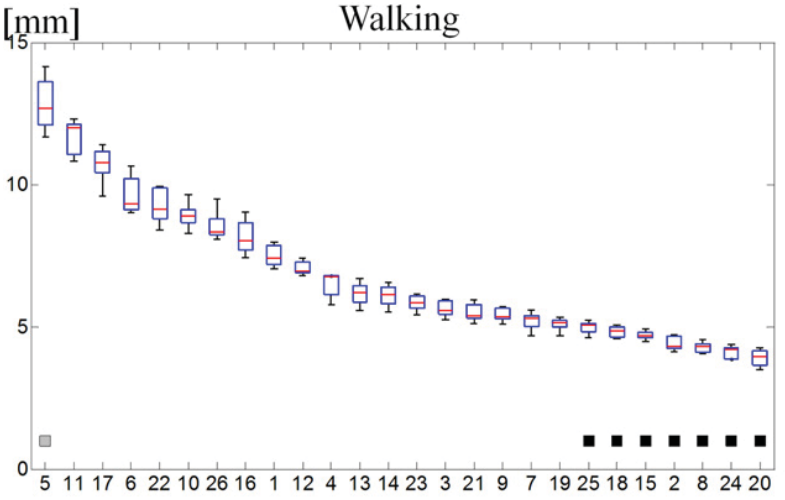

Markers [mm] Landing

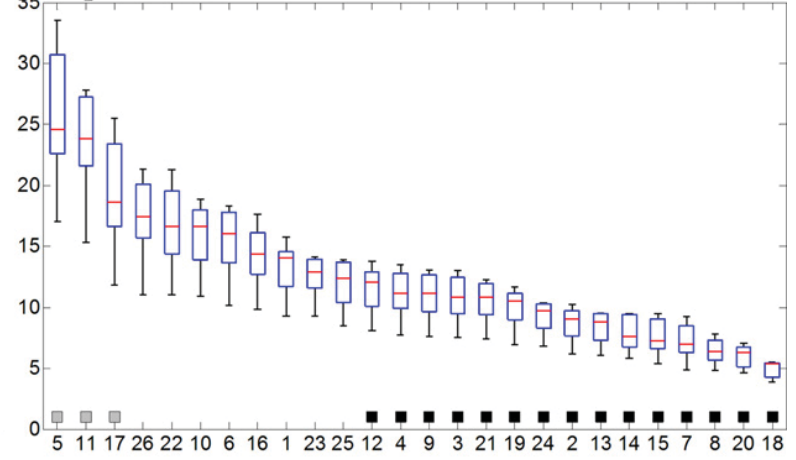

Markers

Fig. B6. Marker deformations for walking and landing for subject 7. Black squares reveal markers that gave the same deformation as the least deformed and grey squares reveal markers that gave the same deformation as the most deformed.

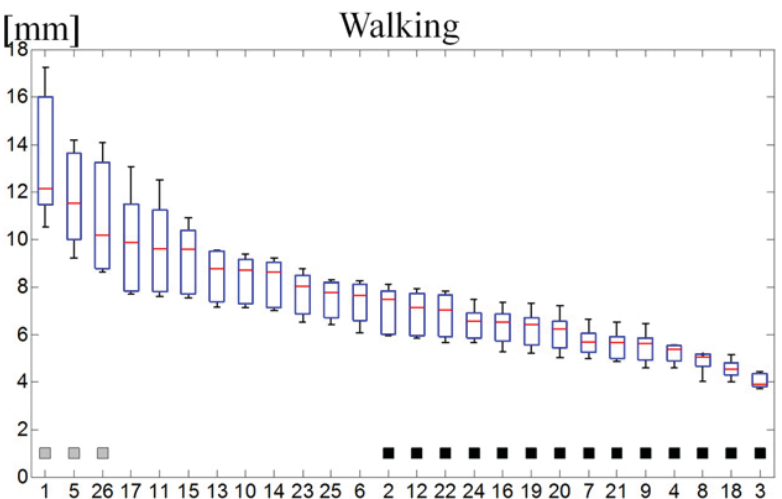

Markers [mm] Landing

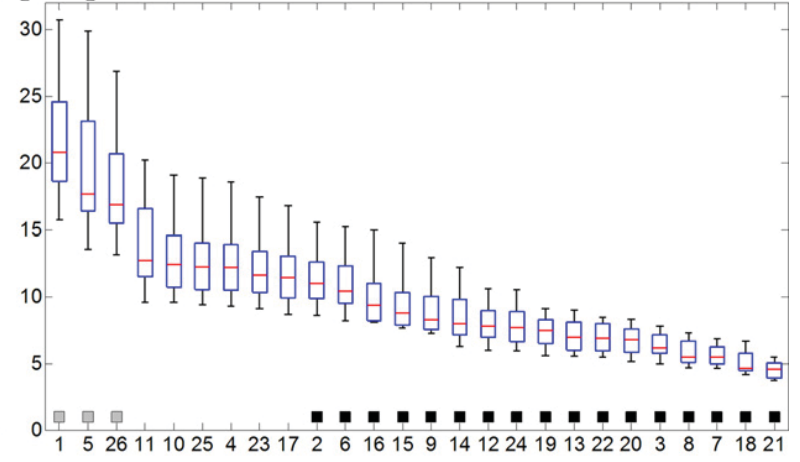

Markers

Fig. B7. Marker deformations for walking and landing for subject 8. Black squares reveal markers that gave the same deformation as the least deformed and grey squares reveal markers that gave the same deformation as the most deformed. 